\title{
Single Center Experiences of Conversion from Twice-daily Tacrolimus (Prograf) to Once-daily Tacrolimus (Advagraf) in Stable Liver Transplant Recipients
}

\author{
Tae-Seok Kim, M.D., Keun Soo Ahn, M.D., Yong Hoon Kim, M.D., Hyoung Tae Kim, M.D. \\ and Koo Jeong Kang, M.D. \\ Department of Surgery, Keimyung University Dongsan Medical Center, Keimyung University School of Medicine, Daegu, Korea
}

Background: Patient adherence to immunosuppressant regimens after organ transplant is crucial to preserve graft function, and simplifying the regimen improves adherence. In this study, our experience of conversion from twice-daily (b.i.d.) to once-daily (q.d.) tacrolimus (TAC) in stable liver transplant recipients is reviewed and the proper conversion regimen is investigated.

Methods: Between November 2011 and August 2012, the regimen was converted in 32 stable liver transplant recipients, and data on the conversions gathered retrospectively from medical records. TAC trough level, dose, and laboratory findings were evaluated at preconversion and 1 to 12 months after conversion.

Results: Conversion from b.i.d. to q.d. regimen was based on 1:1 proportion in 16 patients and dose escalation in 16 patients. The mean conversion time after transplant was 56.8 months (range; 21 94). Reconversion to b.i.d. regimen was needed in nine patients. Among these patients, seven patients needed titration due to elevated liver enzyme. The trough level decreased significantly after conversion (from 4.7 to $3.1 \mathrm{ng} / \mathrm{mL}$ ) in patients with conversion at 1:1 proportion, while increasing slightly without statistical significance ( 3.7 to $4.0 \mathrm{ng} / \mathrm{mL}$ ) in patients with dose escalation. At 1 year after conversion, dose adjustment was required to preserve trough level and graft function in 14 patients.

Conclusions: Based on our results, TAC q.d. formulation can be a useful option to improve adherence in stable liver transplant recipients. However, dose titration should be considered for preserving proper trough level in case of low TAC level or TAC single regimen.

Key Words: Tacrolimus, Immunosuppression, Once-daily tacrolimus 중심 단어: 타크로리무스, 면역억제요법, 하루 1 회 타크로리무스

Received January 5, 2016

Revised June 9, 2016

Accepted June 9, 2016

\section{Corresponding author: Koo Jeong Kang}

Division of Hepatobiliary and Pancreatic Surgery, Department of Surgery, Keimyung University Dongsan Medical Center, Keimyung University School of Medicine, 56 Dalseong-ro, Jung-gu, Daegu 41931, Korea

Tel: 82-53-250-7655, Fax: 82-53-250-7322

E-mail: kjkang@dsmc.or.kr

\section{INTRODUCTION}

Immune tolerance research to withdraw or minimize immunosuppressive drugs after solid organ transplantation is an active field. However, life-long intake of immunosuppressive drugs is essential to preserve graft function, and non-compliance remains a major problem after solid organ transplantation, leading to increased rejection episodes and graft $\operatorname{loss}(1,2)$.

Tacrolimus (TAC) is the major immunosuppressive drug in solid organ transplantation. Before the introduction of once-daily (q.d.) formulation, TAC was available as an im- 
mediate-release formulation administered twice-daily (b.i.d.) to prevent allograft rejection. A prolonged-release formulation TAC administered q.d. in the morning provides more consistent exposure, improves patient adherence(3), and improves therapeutic efficacy, safety, and adherence in patients with solid organ transplants(3-8).

In this study, we review our experience of conversion from TAC b.i.d. to TAC q.d. formulation in stable liver transplant (LT) recipients, evaluate the safety and feasibility of conversion to TAC q.d. formulation, and examine the proper method of conversion, through level, liver function and kidney function.

\section{MATERIALS AND METHODS}

From November 2011 to August 2012, TAC b.i.d. regimen was converted to TAC q.d. in 32 patients following LT in Keimyung University Dongsan Medical Center, Daegu, Korea. All patients in this study fulfilled the following criteria: at least 12 months' posttransplant follow-up, no rejection episode in the last 6 months, and stable liver function and TAC trough level in the last 6 months. The conversion from TAC b.i.d. to TAC q.d. was performed with 1:1 dose ratio in 16 patients and with modified dose escalation of $0.5 \mathrm{mg}$ or $1 \mathrm{mg}$ based on TAC dose in 16 patients. Conversion dose was decided based on trough level at the time of conversion and dose adjustment for preserving proper trough level after conversion was performed at each visit based on the variation of the serum trough level. The primary end-point of this study was proportion of patients with reconversion to TAC b.i.d. formulation over time. Reconversion to TAC b.i.d. was performed without further dose adjustment in patients with any complications, uncontrolled trough level in spite of dose adjustment and clinically suspected rejection (elevated liver enzyme without other causes). Liver and renal function test, TAC dose, serum trough level, and adverse events at 1, 2, 6, and 12 months after conversion were reviewed. Renal function was evaluated by serum creatinine levels and estimated glomerular filtration rate derived from the Modification of Diet in Renal Disease equation.

Quantitative variables were described with mean \pm standard deviation and ranges. The Wilcoxon test (paired t-test) was used to compare the change of through level of TAC before and after conversion and chi-square test to compare categorical variables. Null hypotheses of no difference were rejected if $P$-values were less than 0.05 . All statistical analysis and graph were made using GraphPad Prism ver. 5.0 for Windows (GraphPad Software, San Diego, CA, USA).

\section{RESULTS}

Patient characteristics and conversion results are shown in Table 1. Among 32 patients enrolled in this study, 24 patients were male and eight were female. The mean age at the time of conversion was 55 years (range; 18 72) and mean time since LT was 56.8 months (range; 21 94). Immunosuppresive therapy at the time of conversion consisted of TAC alone in most patients (94\%); TAC and mycophenolate mofetil in only two patients $(6 \%)$. Liver cirrhosis caused by hepatitis B virus with or without hepatocellular carcinoma was the main indication of LT. Biopsy-proven acute celluar rejection after LT occurred in

Table 1. Patients characteristics at the time of conversion $(n=32)$

\begin{tabular}{lc}
\hline \multicolumn{1}{c}{ Characteristic } & Value \\
\hline Sex (males/female) & $24 / 8$ \\
Age at conversion (yr) & $55 \pm 9.9(18 \sim 72)$ \\
Time from LT to conversion & $56.8 \pm 19.49(21 \sim 94)$ \\
Immunosuppressant regimen & 30 \\
TAC single & 2 \\
TAC+MMF & $5(16)$ \\
History of rejection episode & $18(56)$ \\
Indications for LT & $10(31)$ \\
Hepatitis B virus & $4(13)$ \\
Hepatocellular carcinoma & \\
Others & $2.03 \pm 0.60(1 \sim 4)$ \\
Mean TAC dose (mg/day) & $4.2 \pm 1.50(1.9 \sim 7.4)$ \\
Baseline mean trough level (ng/mL) & $9(28)$ \\
Reconversion to TAC twice-daily & $4.4 \pm 4.12(0.9 \sim 11.4)$ \\
Time from conversion to reconversion & \\
Cause of reconversion & $7(5 / 2)$ \\
Elevated AST or ALT (1:1/escalation) & 1 \\
Uncontrolled trough level & 1 \\
Neurologic symptom &
\end{tabular}

Data are presented as mean \pm SD (range) or number (\%). Abbreviations: LT, liver transplant; MMF, mycophenolate mofetil; TAC, tacrolimus; AST, aspartate aminotransferase; ALT, alanine aminotransferase.

${ }^{\mathrm{a}}$ Perioral numbness. 
five patients (16\%) prior to the last 6 months before conversion.

The mean TAC serum through level before conversion was $4.2 \pm 1.5 \mathrm{ng} / \mathrm{mL}$ with a daily dose of $2.0 \pm 0.6 \mathrm{mg}$. Conversion to TAC q.d. was performed with 1:1 ratio dose in 16 patients and with dose escalation in 16 patients. The mean serum TAC trough level decreased significantly by 1 month after conversion in 1:1 ratio conversion group (from 4.7 to $3.1 \mathrm{ng} / \mathrm{mL}, P=0.001$ ), while there was no significant change of serum trough level (from 3.7 to $4.0 \mathrm{ng} / \mathrm{mL}$ ) in the dose escalation group (Fig. 1). During follow-up period, nine patients returned to TAC b.i.d., and 23 patients were still on TAC q.d. without deterioration of liver and kidney

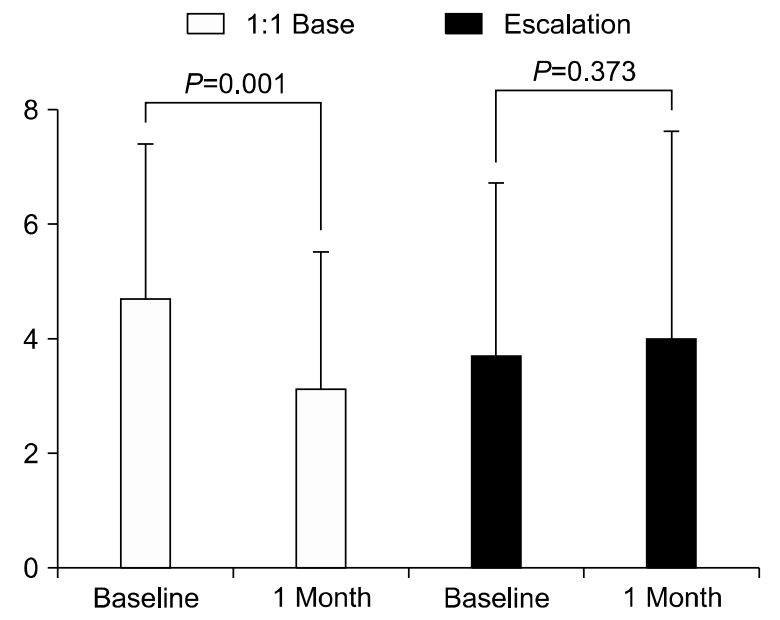

Fig. 1. Trough level change at 1 month after conversion $(n=32)$. function. For these 23 patients with successful conversion, a significantly increased dose of TAC (from 2.1 to 2.6 $\mathrm{mg} /$ day, $P=0.001$ ) was required to maintain the serum trough level (Table 2). The mean time from converion to reconversion was 4.4 months (range; 0.9 11.4). The reconversion from TAC q.d. to TAC b.i.d. was due to elevated liver enzyme $(n=7)$, uncontrolled trough level $(n=1)$, or development of neurologic symptoms $(n=1)$. Comparing the $1: 1$ and dose escalation groups, there was no statistically significant difference in charateristics between groups except conversion dose (Table 3). However, mean trough level at the time of conversion was lower in dose escalation group (3.7 $\mathrm{ng} / \mathrm{mL}$ vs. $4.7 \mathrm{ng} / \mathrm{mL}$ ) than 1:1 group, although not a significant difference due to small number of cases. This is the reason for dose escalation during converison. There were slightly more patients who need reconversion to TAC b.i.d. formulation ( $31 \%$ vs. $13 \%$ ), not a significant difference.

For these seven patients who returned to TAC b.i.d. due to elevated liver enzyme, the mean serum trough level was decreased from $4.9 \mathrm{ng} / \mathrm{mL}$ at the time of conversion to 4.0 $\mathrm{ng} / \mathrm{mL}$ at the time of reconversion and trough level decreased more in five patients in the 1:1 dose group (from 5.0 to $3.8 \mathrm{ng} / \mathrm{mL}$ vs. from 4.5 to $4.4 \mathrm{ng} / \mathrm{mL}$ ). After the reconversion, the mean trough level increased significantly while taking the same daily dose of TAC except one patient (from 4.0 to $5.7 \mathrm{ng} / \mathrm{mL}, P=0.013$ ). After the reconversion to TAC b.i.d., liver function maintained stable levels and symptoms improved in all patients.

Table 2. Liver function, kidney function, serum trough level, and dose of TAC in patients with successful conversion at baseline and after the conversion from TAC twice-daily to TAC once-daily $(n=23)$

\begin{tabular}{lcccccc}
\hline \multirow{2}{*}{ Variable } & \multicolumn{7}{c}{ Time (mo) } & & \\
\cline { 2 - 6 } & Baseline & 1 & 3 & 6 & 12 & \\
\hline ALP (U/L) & $254 \pm 124$ & $246 \pm 115$ & $228 \pm 114$ & $232 \pm 99$ & $235 \pm 105$ & 0.001 \\
AST (U/L) & $22 \pm 6$ & $21 \pm 5$ & $22 \pm 6$ & $22 \pm 5$ & $23 \pm 6$ & 0.727 \\
ALT (U/L) & $21 \pm 12$ & $20 \pm 10$ & $21 \pm 9$ & $22 \pm 10$ & $23 \pm 10$ & 0.819 \\
Total bilirubin (mg/dL) & $0.9 \pm 0.3$ & $0.9 \pm 0.4$ & $1.0 \pm 0.4$ & $1.0 \pm 0.5$ & $0.9 \pm 0.4$ & 0.640 \\
Serum Cr (mg/dL) & $0.9 \pm 0.3$ & $0.9 \pm 0.2$ & $1.0 \pm 0.2$ & $1.0 \pm 0.2$ & $0.9 \pm 0.2$ & 0.178 \\
eGFR (mL/min) & $94 \pm 28$ & $93 \pm 26$ & $87 \pm 24$ & $88 \pm 25$ & $91 \pm 27$ & 0.095 \\
TAC level (ng/mL) & $3.9 \pm 1.4$ & $3.2 \pm 1.5$ & $3.4 \pm 1.5$ & $3.6 \pm 1.1$ & $3.8 \pm 1.6$ & 0.254 \\
TAC dose (mg) & $2.1 \pm 0.6$ & $2.4 \pm 0.8$ & $2.5 \pm 0.8$ & $2.5 \pm 0.8$ & $2.6 \pm 0.8$ & 0.001 \\
\hline
\end{tabular}

Data are presented as mean $\pm \mathrm{SD}$.

Abbreviations: TAC, tacrolimus; ALP, alkaline phosphatase; AST, aspartate aminotransferase; ALT, alanine aminotransferase; eGFR, estimated glomerular filtration rate. 
Table 3. Comparison of characteristics and results between 1:1 dose group and escalation group

\begin{tabular}{lccc}
\hline \multicolumn{1}{c}{ Characteristic } & $1: 1$ Group & Escalation group & $P$-value \\
\hline Sex (male/female) & $12 / 4$ & $12 / 4$ & 0.577 \\
Age at conversion (yr) & $54 \pm 11.6$ & $56 \pm 8.1$ & 0.926 \\
Time from LT to conversion (mo) & $56.5 \pm 23.4$ & $57.2 \pm 15.3$ & 0.632 \\
History of rejection & $3(19)$ & $2(13)$ & 0.772 \\
Mean TAC dose (mg/day) & $2.0 \pm 0.5$ & $2.1 \pm 0.7$ & 0.013 \\
Mean conversion dose (mg/day) & $2.0 \pm 0.5$ & $2.7 \pm 0.8$ & 0.056 \\
Baseline mean trough level (ng/mL) & $4.7 \pm 1.5$ & $3.7 \pm 1.4$ & 0.110 \\
Mean trough level after conversion (ng/mL) & $3.1 \pm 1.3$ & $4(25)$ & 0.699 \\
Reconversion to TAC twice-daily & $5(31)$ & $2(13)$ & 0.207 \\
Reconversion due to elevated AST or ALT & $5(31)$ & \\
\hline
\end{tabular}

Data are presented as mean $\pm \mathrm{SD}$ or number $(\%)$.

Abbreviations: LT, liver transplant; TAC, tacrolimus; AST, aspartate aminotransferase; ALT, alanine aminotransferase.

\section{DISCUSSION}

Drug adherence declines over time in patients after organ transplantation and depends on the type of medication, the number of drugs and daily doses(9). With b.i.d. formulation, adherence appears significantly lower for the evening versus the morning dose(10). Introduction of TAC q.d. formulation, administered in the morning, is associated with better adherence and improved graft survival(4,6,11,12). The safety and efficacy of TAC q.d. formulation after solid organ transplantation was reported by many groups. In our study, TAC q.d. formulation seems a useful therapeutic option in most patients, which can improve adherence to immunosuppressive therapy. However, reconversion rate was about $28 \%$ and higher than reported in the literature. One of the causes of higher reconversion rate was immediate reconversion to TAC b.i.d. without biopsy or dose adjustment when liver enzymes became elevated. In these patients, the mean trough level decreased significantly after conversion and increased after reconversion despite the same daily dose of TAC b.i.d., and the liver enzyme stablized after reconversion. This trend was more frequently observed in 1:1 dose group. Based on this result, we suggest that dose titration based on serum trough level could improve conversion to TAC q.d. formulation.

Decreased TAC trough level after a 1:1 conversion in stable LT recipients was reported by several authors $(3,12-14)$. We also observed significantly decreased TAC trough levels after a 1:1 conversion and dose escalation was required to maintain TAC trough level in our study. Dopazo et al.(13) reported that TAC trough level decreased approximately $20 \% 1$ month after conversion and reached initial value 6 to 12 months after conversion without dose adjustment in most patients $(88 \%)$. However, dose adjustment was required to maintain proper TAC trough level in our study. Sanko-Resmer et al.(14) included 112 stable LT recipients converted formulation with $1: 1$ ratio in whom the mean TAC trough level was reduced by $15 \%$ without any acute rejection, and remained stable $(74.5 \%$ of the patients required no dose adjustment on conversion). In our study, $52 \%$ of the patients with stable liver function after conversion later required dose adjustment maintain TAC trough level and stable liver function. We suppose that these results were caused by the differences in immunosuppressant regimen and baseline TAC level. In our study, the TAC single regimen was adopted in most patients, and the baseline trough level was lower than in other studies due to minimization of TAC dose. Thus, dose adjustment was performed in more patients.

Seven patients (22\%) developed elevated liver enzyme and required reconversion (although the acute rejection was not proven by biopsy despite elevated liver enzyme). In these patients, decreased trough level after conversion was observed and this trend was more definite in the 1:1 dose group. Therefore, we suggest that initial dose escalation of $\mathrm{TAC}$ or close monitoring and maintaining of trough level by adjustment of TAC dose could be helpful for successful conversion to TAC q.d. formulation in patients with low 
TAC level.

However, our study has some limitations. First, our study is retrospective with a very small sample size. Therefore it is hard to generalize our result. Second, in patients with elevated liver enzyme, reconversion to TAC b.i.d. formulation was performed immediately without dose adjustment or biopsy. Therefore the reconversion rate in our study was higher than other studies, and our study is limited on the investigation into the effect of TAC q.d. formulation on rejection. So, further study, well-designed with a large sample size will be needed to confirm our result.

\section{CONCLUSION}

The theapeutic equivalence of TAC q.d. compared to TAC b.i.d. is supported strongly by many studies, and our study showed feasible results after conversion to TAC q.d. formulation. Therefore, TAC q.d. formulation is a useful option for effective immunosuppressive therapy and improving adherence after LT. However, dose escalation and adjustment after close monitoring of trough level could be required when TAC level maintains low or single TAC regimen is applied.

\section{REFERENCES}

1) Chapman JR. Compliance: the patient, the doctor, and the medication? Transplantation 2004;77:782-6.

2) Lieber SR, Volk ML. Non-adherence and graft failure in adult liver transplant recipients. Dig Dis Sci 2013;58:824-34.

3) Dumortier J, Guillaud O, Boillot O. Conversion from twice daily tacrolimus to once daily tacrolimus in long-term stable liver transplant recipients: a single-center experience with 394 patients. Liver Transpl 2013;19:529-33.

4) Beckebaum S, Iacob S, Sweid D, Sotiropoulos GC, Saner F, Kaiser G, et al. Efficacy, safety, and immunosuppressant adherence in stable liver transplant patients converted from a twice-daily tacrolimus-based regimen to once-daily tacrolimus extended-release formulation. Transpl Int 2011;
24:666-75.

5) Alloway RR, Eckhoff DE, Washburn WK, Teperman LW. Conversion from twice daily tacrolimus capsules to once daily extended-release tacrolimus (LCP-Tacro): phase 2 trial of stable liver transplant recipients. Liver Transpl 2014;20: 564-75.

6) Giannelli V, Rossi M, Giusto M, Lucidi C, Lattanzi B, Ruffa A, et al. Conversion from twice-daily to once-daily Tacrolimus administration in liver transplant patient: results of long term follow-up. Eur Rev Med Pharmacol Sci 2013; 17:2718-20.

7) Valente G, Rinaldi L, Sgambato M, Piai G. Conversion from twice-daily to once-daily tacrolimus in stable liver transplant patients: effectiveness in a real-world setting. Transplant Proc 2013;45:1273-5.

8) Jannot M, Masson I, Alamartine E, Mariat C. Early conversion from twice-daily tacrolimus to once-daily extended formulation in renal transplant patients before hospital discharge. Ann Transplant 2014;19:320-4.

9) Laederach-Hofmann K, Bunzel B. Noncompliance in organ transplant recipients: a literature review. Gen Hosp Psychiatry 2000;22:412-24.

10) Ichimaru N, Kakuta $Y$, Abe T, Okumi M, Imamura R, Isaka $\mathrm{Y}$, et al. Treatment adherence in renal transplant recipients: a questionnaire survey on immunosuppressants. Transplant Proc 2008;40:1362-5.

11) Trunecka $P$, Boillot $O$, Seehofer $D$, Pinna $A D$, Fischer $L$, Ericzon BG, et al. Once-daily prolonged-release tacrolimus (ADVAGRAF) versus twice-daily tacrolimus (PROGRAF) in liver transplantation. Am J Transplant 2010;10:2313-23.

12) Kim SH, Lee SD, Kim YK, Park SJ. Conversion of twice-daily to once-daily tacrolimus is safe in stable adult living donor liver transplant recipients. Hepatobiliary Pancreat Dis Int 2015;14:374-9.

13) Dopazo C, Rodriguez R, Llado L, Calatayud D, Castells L, Ramos E, et al. Successful conversion from twice-daily to once-daily tacrolimus in liver transplantation: observational multicenter study. Clin Transplant 2012;26:E32-7.

14) Sanko-Resmer J, Boillot $O$, Wolf $P$, Thorburn D. Renal function, efficacy and safety postconversion from twice- to once-daily tacrolimus in stable liver recipients: an open-label multicenter study. Transpl Int 2012;25:283-93. 